\title{
Anisotropic Ellipsoidal Smoothing of Volume Data
}

\author{
Bai Xue ${ }^{1}$, Nelson Max ${ }^{1,2}$, Cristina Siegerist ${ }^{2}$ \\ ${ }^{1}$ University of California, Davis ${ }^{2}$ Lawrence Berkeley Laboratory
}

\begin{abstract}
:
This paper describes a simple image processing algorithm for identifying and smoothing cell membranes in tomographic reconstructions of electron micrographs of frozen bacteria.
\end{abstract}

CR Categories and Subject Descriptors: I.4.3 filtering

Additional Keywords: anisotropic filter, ellipsoidal filter

\section{INTRODUCTION}

The goal of this research was the visualization of bacterial cell membranes in cryo-electron microscope reconstructions. The bacteria cell density data was obtained from a JEOL-3100-SFF electron microscope with a FEG electron source operating at 300 $\mathrm{kV}$. By tilting the microscope stage between exposures, tilt series were acquired with an average angular range between approximately $+65 \mathrm{deg}$ and $-65 \mathrm{deg}$, with increments of $1.5 \mathrm{deg}$ or 2 deg. Between 70 and 90 images were recorded for each series. All data sets were acquired using a Digital Micrograph (Gatan). The IMOD package [1] was used for image processing and tomographic reconstructions by back projection. Figure 1 shows an electron micrograph from a tilt series, and a slice through its reconstruction.
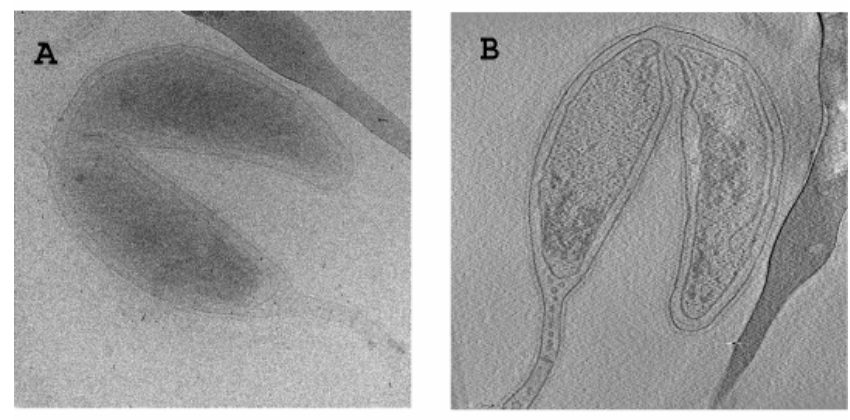

Figure 1. A: An electron micrograph from a tilt series. B a slice through the tomographic reconstruction.

Because high electron doses can destroy the specimen before the tilt series is complete, the original micrographs were of low dose, and therefore noisy. The tomographic reconstruction process removes some of this noise, but much remains. In addition, the restricted angular range in which the specimen can be tilted means that some views which would aid in the tomographic reconstruction are unavailable, making the cell membrane harder to distinguish in certain surface orientations. The membrane is characterized by thickened surface-like regions of high density, but has gaps due to the noise and poor reconstruction. Therefore, we attempted to determine a filtering process that could smooth across gaps, before using iso-contouring.

Recent anisotropic filtering techniques using partial differential equations can smooth contiguous noisy regions without destroy-

${ }^{1}$ Univ. of CA, Davis, One Shields Ave., Davis, CA 95616, USA

bxue@ucdavis.edu,max2@1nl.gov, cristina@hpcrd.lbl.gov ing the sharp edges between them [2, 3, 4, 5]. They are appropriate for removing noise before edge detection. However, they are not as useful for filling gaps in surface-like high density regions. We developed a simple filter specifically tailored for this purpose. Briefly, we predicted the surface normal direction, and then used oblate (flattened) ellipsoidal filters, whose short axis were oriented along the predicted normal, so as not to blur the surface thickness, and whose longer axes were oriented tangent to the surface, to blur across the gaps.

\section{SURFACE NORMAL ESTIMATION}

We used principal component analysis to estimate the surface normal from the covariance matrix. If a point lies near a surface, we expect that in a neighborhood of the point, the density will be well represented by a thickened plane, and the normal to this plane can be estimated as the eigenvector of the covariance matrix with the smallest eigenvalue.

The cell is surrounded by ice, with a density that is only slightly lower than that of the membrane. For a more effective principal component analysis, we subtracted an estimate of the ice density (currently the median density value), and set any negative values to zero. We then could use cubical box windows for the voxel neighborhoods, without introducing much extra bias in the normal estimates, compared to spherical windows. An advantage of the cubical boxes is that the sums used in estimating the covariance matrix from which principal components are calculated can be computed in a separable manner. For example, if the inner loop in such an $n$ by $n$ by $n$ sum runs over the $z$ direction, this sum can be reused for all the $n^{2}$ voxels whose windows contain exactly this $z$ segment, saving a factor of $n^{2}$ in the computation.

In the computations below, all sums are over the voxel's cubical box neighborhood. The data value at voxel $(x, y, z)$ is $v(x, y, z)$. The center of gravity is

$$
(\bar{x}, \bar{y}, \bar{z})=\frac{\sum v(x, y, z)(x, y, z)}{\sum v(x, y, z)}
$$

and the covariance matrix is

$$
M=\left(\begin{array}{lll}
M_{x x} & M_{x y} & M_{x z} \\
M_{y x} & M_{y y} & M_{y z} \\
M_{z x} & M_{z y} & M_{z z}
\end{array}\right)
$$

where

$$
\begin{aligned}
& M_{x x}=\sum v(x, y, z)(x-\bar{x})(x-\bar{x}), \\
& M_{y y}=\sum v(x, y, z)(y-\bar{y})(y-\bar{y}), \\
& M_{z z}=\sum v(x, y, z)(z-\bar{z})(z-\bar{z}), \\
& M_{x y}=M_{y x}=\sum v(x, y, z)(x-\bar{x})(y-\bar{y}), \\
& M_{y z}=M_{z y}=\sum v(x, y, z)(y-\bar{y})(z-\bar{z}), \\
& M_{x z}=M_{z x}=\sum v(x, y, z)(x-\bar{x})(z-\bar{z}) .
\end{aligned}
$$


We expect that for $M$ the smallest eigenvalue's eigenvector should be normal to the surface, as shown in 2D in Figure 2. Thus we can choose an anisotropic ellipsoidal filter, which smoothes more along the surface than in the normal direction.

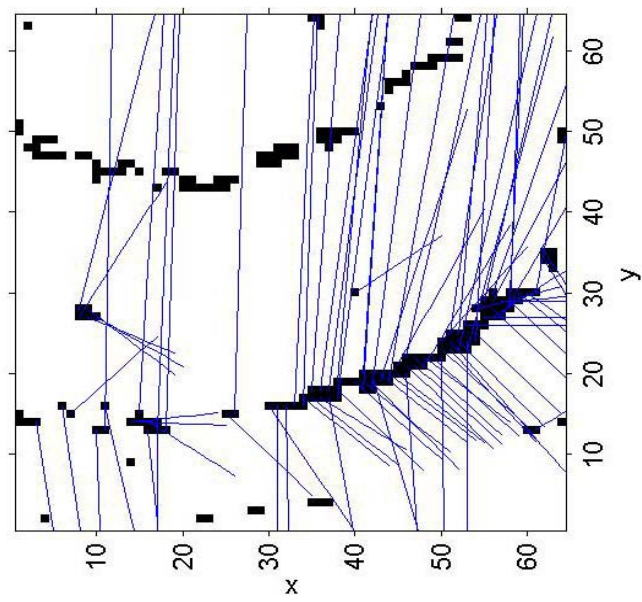

Figure 2. 2D section of a membrane. Black membrane pixels were selected by thresholding, erosion, any dilation. Blue lines show projected eigenvectors to the smallest eigenvalues of the lower part of the membrane

\section{Ellipsoidal Filter CONSTRUCtion}

Let $v_{1}, v_{2}, v_{3}$ be the three eigenvectors of the matrix $M$, represented as column vectors and in order of decreasing eigenvalue, so that $v_{3}$ is the one with the smallest eigenvalue. Define a rotation matrix by $R=\left(v_{1}, v_{2}, v_{3}\right)^{T}$ with $R^{T}=\left(v_{1}, v_{2}, v_{3}\right)$.

We construct the filter by rotating an axis aligned ellipsoidal Gaussian filter represented by a diagonal matrix A, by the rotation matrix $\mathrm{R}$, to align its axes to the eigenvector directions found above. For a filter box centered at $\left(x_{0}, y_{0}, z_{0}\right)$, the filter weights are

$$
W(x, y, z)=e^{-\left(x-x_{0}, y-y_{0}, z-z_{0}\right) R^{T} A R}\left(\begin{array}{l}
x-x_{0} \\
y-y_{0} \\
z-z_{0}
\end{array}\right)
$$

where

$$
A=\left(\begin{array}{ccc}
\frac{1}{r_{1}^{2}} & 0 & 0 \\
0 & \frac{1}{r_{2}^{2}} & 0 \\
0 & 0 & \frac{1}{r_{3}^{2}}
\end{array}\right),
$$

and $r_{1}, r_{2}, r_{3}$ are the filter radii in $x, y, z$ and orientation, with $r_{1}=r_{2}>r_{3}$. In the figures below, $r_{1}=r_{2}=7$ and $r_{3}=2$ or 3 .

The voxel values are smoothed by this filter as follows:

$$
S D\left(x_{0}, y_{0}, z_{0}\right)=\frac{\sum W\left(x-x_{0}, y-y_{0}, z-z_{0}\right) v(x, y, z)}{\sum W\left(x-x_{0}, y-y_{0}, z-z_{0}\right)}
$$

where $S D\left(x_{0}, y_{0}, z_{0}\right)$ is the smoothed data in the center of the box. Note that the normalization by the sum of the filter weights eliminates the need for the usual normalization factor in Formula (3), and the need to account for the clipped portion of the Gaussian filter that extends outside the filter box.

Figure 3 shows a slice through a volume reconstructed by tomography. Figure 4 shows the same slice on a volume that has been filtered by an oriented $7 \times 7 \times 2$ ellipsoidal filter. Note that the two adjacent dark membranes are now much clearer and less noisy. Figure 5 shows contour surfaces for the results of using an anisotropic $3 \times 3 \times 3$ filter, and figure 6 , which has a considerable smoother surface, shows the results of using our $7 \times 7 \times 3$ ellipsoidal filter, oriented by the estimated normal as discussed above. Both filters used an $11 \times 11 \times 11$ box, as did the computations of the normal estimate in equations (1), (2), and (3). On a data set of size $208 \times 208 \times 102$, both filters took approximately 18 minutes in a C implementation without the speed-ups from the separable box, on an AMD Opteron 1992.665 MHz CPU, with an 8 Gbyte RAM.

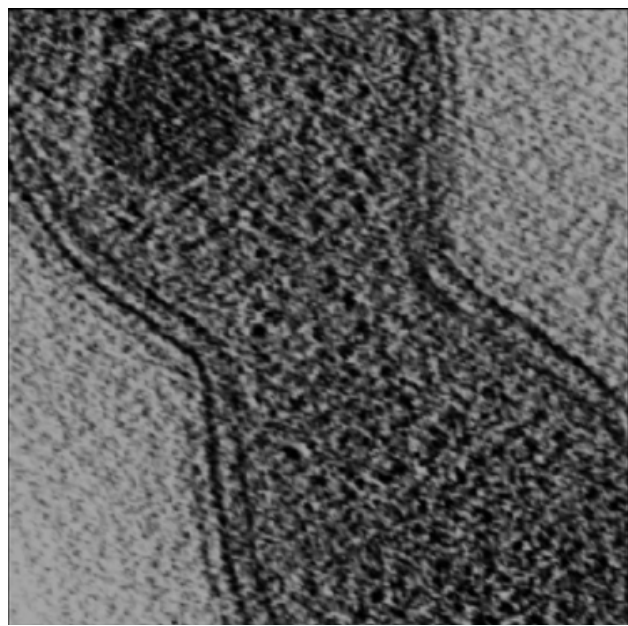

Figure 3. Slice of original density data

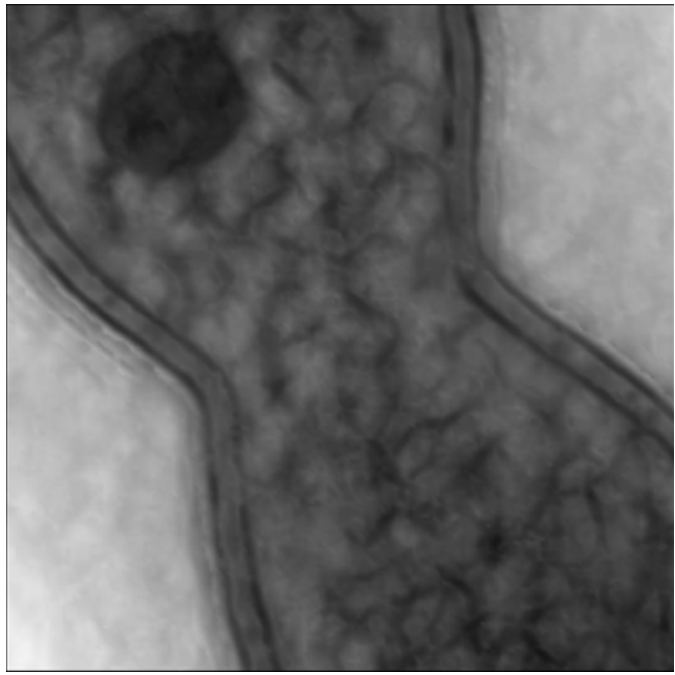

Figure 4. Slice of data after $7 \times 7 \times 2$ ellipsoidal filtering

\section{REGION GROWING}

Figures 5 and 6 were made using a fairly low contour level, and show a surface near the exterior of the cell. There are two adjacent membranes around a bacterial cell, and a contour level which can detect both will also detect many other sub-cellular structures with similar density. Therefore we use the region growing software of Huang et al. [6]. The user selects a seed voxel on a chosen 2D section, and the region is grown by selecting for inclusion among the 26 neighbors, based on similarity in scalar value and gradient magnitude to the seed voxel. Figure 7 the result of region growing for a cell after isotropic filtering. Figure 8 shows the region growing algorithm plus extra smoothing applied to the same data 
after the anisotropic filtering discussed above. Note that the surfaces have fewer gaps and extend farther. The ellipsoidal filtering was done in a MATLAB version of the code on a PC with a $3.06 \mathrm{GHz}$ Pentium $4 \mathrm{CPU}$ and $256 \mathrm{M}$ RAM. For this $200 \mathrm{x}$ $220 \times 112$ voxel data set, the filtering took 20.25 minutes. The region growing algorithm, when applied to the data set in figures 3 through 6 , produced the output in figure 9 .

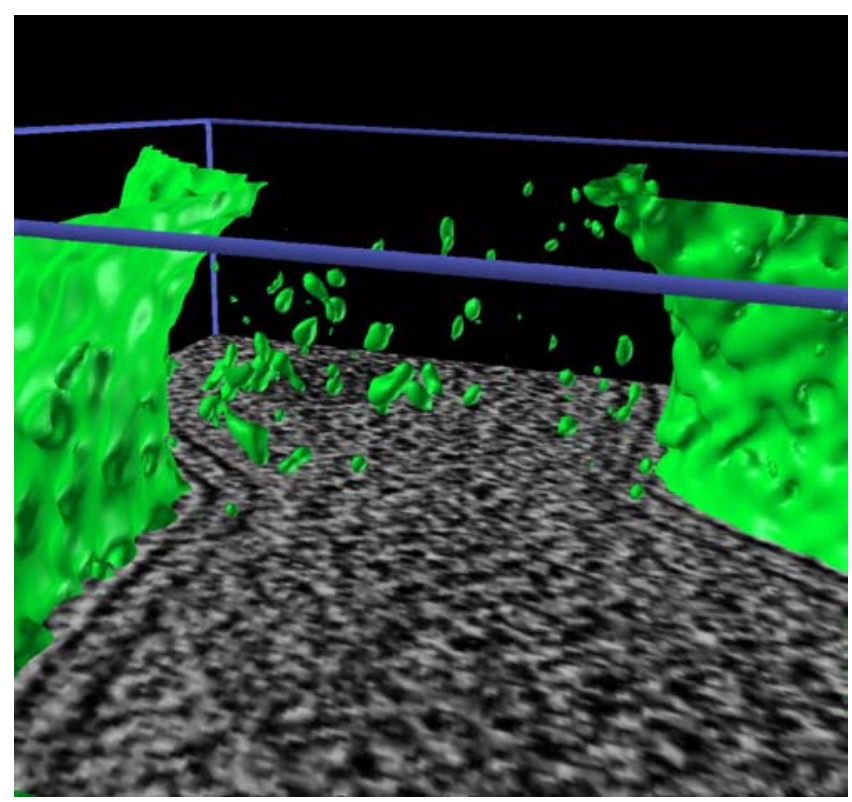

Figure 5. Contour surface from $3 \times 3 \times 3$ filtered data.

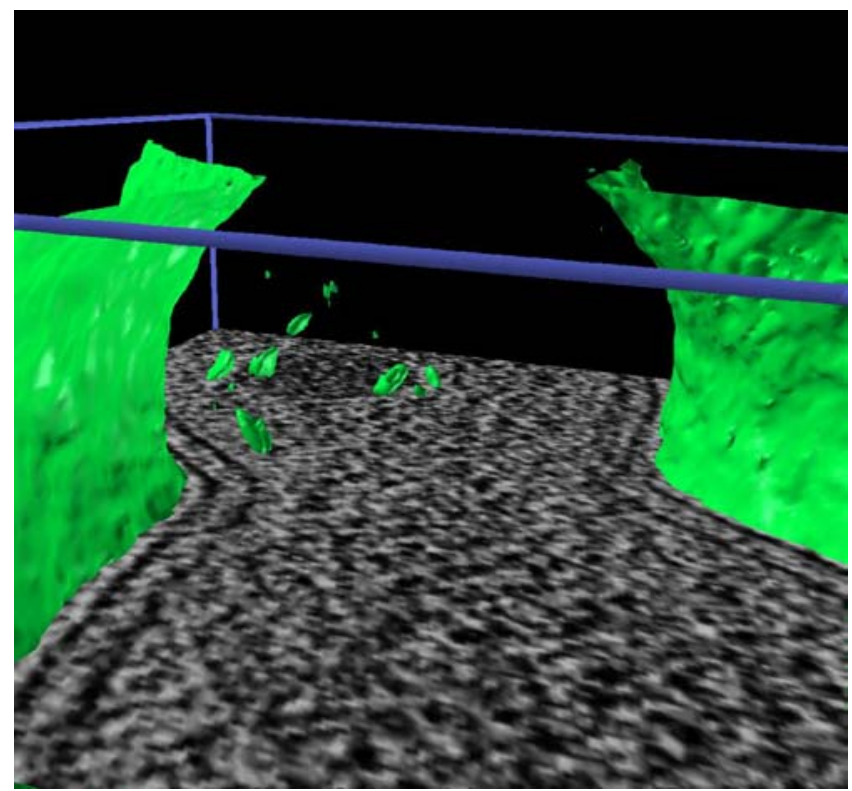

Figure 6. Contour surface from oriented $7 \times 7 \times 3$ filter.

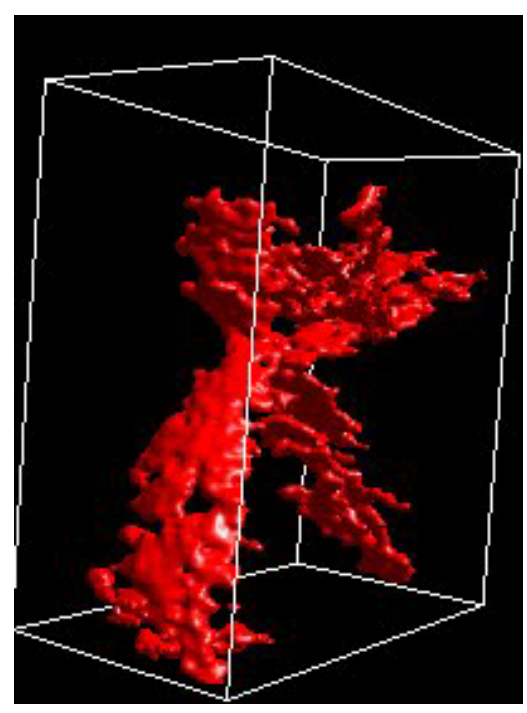

Figure 7. Region growing applied after a $3 \times 3 \times 3$ isotropic filter.

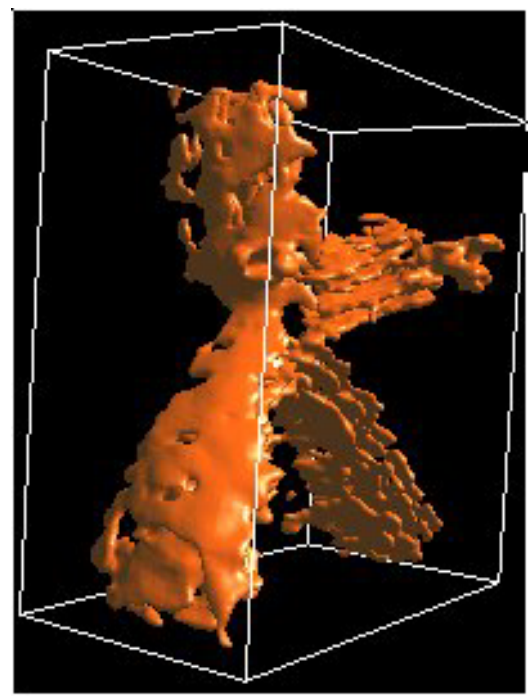

Figure 8. Region growing applied after a $7 \times 7 \times 3$ anisotropic filter.

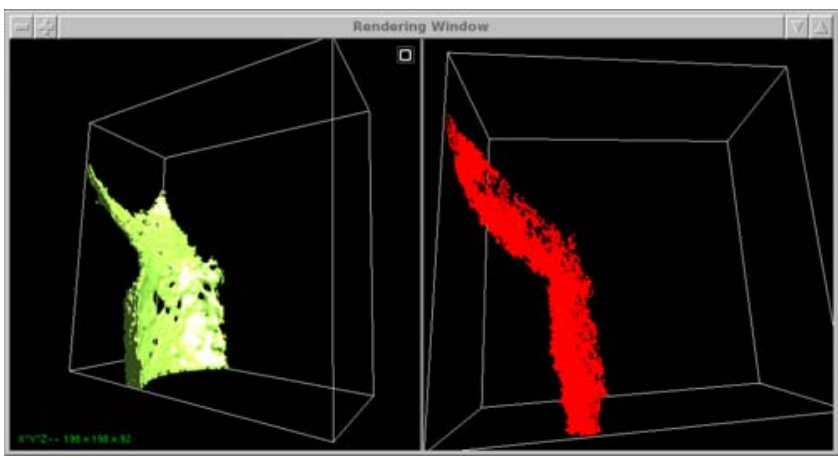

Figure 9. Region growing applied to smooth volume in figure 6. Shaded surface on the left and polygonal mesh on the right. 


\section{SUMMARY}

In order to detect and smooth cell membrane surfaces in tomographic reconstructions of bacteria, we have constructed adaptively oriented anisotropic ellipsoidal filters, which are wide along the surface directions, to smooth noise and suppress gaps, and narrow normal to the surface, in order not to blur it. The surface normal is estimated by principal component analysis. This filtering improves subsequent isocontour or region growing output.

\section{ACKNOWLEDGEMENT}

This work was supported by the Director, Office of Science, Office of Advanced Scientific Computing Research, of the U.S. Department of Energy under Contract No. DE-AC03-76SF00098. Runzhen Huang supplied to us the source code for RGVis. The electron micrographs and their tomographic reconstructions were supplied by Luis Comolli.

\section{REFERENCES}

[1] Kremer JR, Mastronarde DN, McIntosh JR. Computer visualization of three-dimensional image data using IMOD. J Struct Biol. 1996 Jan-Feb; 116(1): 71-6.

[2] Perona P. and Malik J. Scale space and edge detection using anisotropic diffusion. IEEE Transactions on Pattern Analysis and Machine Intelligence, 12(7): 629-639, 1990.

[3] Gerig G., Kubler O., Kikinis R., and Jolesz F. A. Nonlinear anisotropic filtering of MRI data. IEEE Transactions on Medical Imaging, 11(2): 221-232, 1992.

[4] Yang G. Z., Burger P., Firmin D. N., and Underwood S. R. Structure adaptive anisotropic filtering for magnetic resonance images. Lecture Notes in Computer Science, 970:384-391, 1995

[5] Jan-Mark Geusebroek, Arnold W. M. Smeulders, and Joost van de Weijer. Fast anisotropic Gauss filter. IEEE Transactions on Image Processing, 12(8): 938-943, 2003

[6] Runzhen Huang, Kwan-Liu Ma. RGVis: Region Growing Based Techniques for Volume Visualization, Proceedings of the Pacific Graphics 2003 Conference, 355-363, 2003 